University of Nebraska - Lincoln

DigitalCommons@University of Nebraska - Lincoln

Nutrition and Health Sciences -- Faculty

Publications

Nutrition and Health Sciences, Department of

2014

\title{
Gastrointestinal Problems in Children with Autism, Developmental Delays or Typical Development
}

\author{
Virginia Chaidez \\ University of Nebraska - Lincoln, vchaidez2@unl.edu \\ Robin L. Hansen \\ University of California, Davis School of Medicine, rlhansen@ucdavis.edu \\ Irva Hertz-Picciotto \\ University of California, Davis, iher@ucdavis.edu
}

Follow this and additional works at: https://digitalcommons.unl.edu/nutritionfacpub

Part of the Human and Clinical Nutrition Commons, Molecular, Genetic, and Biochemical Nutrition Commons, and the Other Nutrition Commons

Chaidez, Virginia; Hansen, Robin L.; and Hertz-Picciotto, Irva, "Gastrointestinal Problems in Children with Autism, Developmental Delays or Typical Development" (2014). Nutrition and Health Sciences -- Faculty Publications. 232.

https://digitalcommons.unl.edu/nutritionfacpub/232

This Article is brought to you for free and open access by the Nutrition and Health Sciences, Department of at DigitalCommons@University of Nebraska - Lincoln. It has been accepted for inclusion in Nutrition and Health Sciences -- Faculty Publications by an authorized administrator of DigitalCommons@University of Nebraska Lincoln. 


\title{
Gastrointestinal Problems in Children with Autism, Developmental Delays or Typical Development
}

\author{
Virginia Chaidez, ${ }^{1}$ Robin L. Hansen, ${ }^{2,3}$ \\ \& Irva Hertz-Picciotto 3,4
}

1 UC CalFresh Nutrition Education Program, State Office, University of California, Davis

2 Department of Pediatrics, School of Medicine, University of California, Davis

3 Medical Investigations of Neurodevelopmental Disorders (MIND) Institute, University of California, Davis

4 Division of Environmental and Occupational Health, Department of Public Health Sciences, University of California, Davis

Correspondence: V. Chaidez, University of Nebraska-Lincoln, vchaidez2@unl.edu

\begin{abstract}
To compare gastrointestinal (GI) problems among children with: (1) autism spectrum disorder (ASD), (2) developmental delay (DD) and (3) typical development (TD), Gl symptom frequencies were obtained for 960 children from the CHildhood Autism Risks from Genetics and Environment (CHARGE) study. We also examined scores on five Aberrant Behavior Checklist $(A B C)$ subscales comparing ASD children with high versus low frequency $\mathrm{Gl}$ symptoms. Compared to TD children, those with ASD [aOR 7.92 (4.89-12.85)] and DD [aOR 4.55 (2.51-8.24)] were more likely to have at least one frequent Gl symptom. Restricting to ASD children, those with frequent abdominal pain, gaseousness, diarrhea, constipation or pain on stooling scored worse on irritability, social withdrawal, stereotypy, and hyperactivity compared with children having no
\end{abstract}

Published in Journal of Autism and Developmental Disorders (2014) 44:1117-1127 DOI: $10.1007 /$ s10803-013-1973-x

Copyright (c) 2013 Springer Science+Business Media New York. Used by permission. Published 6 November 2013. 
frequent GI symptoms. Frequent GI problems affect young children with ASD and DD more commonly than those with TD. Maladaptive behaviors correlate with GI problems, suggesting these comorbidities require attention.

Keywords: Gastrointestinal problems, Autism, Developmental delays, Maladaptive behaviors

\section{Introduction}

Frequent anecdotal reports of gastrointestinal (GI) problems in children with autism spectrum disorder (ASD) are beginning to be clarified by research efforts examining the issue. The connection between Gl problems and autism is not yet resolved, however, and a handful of recent reports provide conflicting findings, where prevalence of GI symptoms ranges from 23 to $70 \%$ (Molloy and Manning- Courtney 2003; Valicenti-McDermott et al. 2006; Ibrahim et al. 2009; Nikolov et al. 2009; Wang et al. 2011; Gorrindo et al. 2012). Variations in prevalence of Gl problems in large part may be due to differences across studies including, but not limited to: variations in the criteria used to define a GI problem, the number of different GI symptoms considered, the definition of any particular Gl symptom or lack thereof, variations in methodology such as data source (medical chart versus selfreport) or time period for reporting (last few months, lifetime, etc.), and study population characteristics such as age and other criteria for participation.

Research using large samples has begun to provide a better understanding of the heterogeneity of GI concerns in children with ASD (Molloy and Manning-Courtney 2003; Valicenti-McDermott et al. 2006; Ibrahim et al. 2009; Nikolov et al. 2009; Wang et al. 2011). However, for comparative purposes, few estimates of $\mathrm{Gl}$ symptom prevalence are available for children with typical development (TD). Instead, research has focused on Gl issues for groups that seem disproportionately affected, namely children with ASD, and more recently, children with developmental disabilities (Schieve et al. 2012). Among the first of these was a study $(n=137)$ using medical records from a clinic specializing in ASD in a large pediatric medical center serving a 10 county catchment area in the Midwest (Molloy and Manning-Courtney 2003). The study considered solely four Gl symptoms and the definitions were relatively restrictive, which may partially explain prevalence of $24 \%$ in this study population. Nikolov and colleagues evaluated GI problems 
in a sample $(n=172)$ of children with pervasive developmental disorders (PDDs) enrolled for one of two randomized clinical trials. They defined a GI problem as one that caused impairment in function, had been brought to the attention of a medical professional and had been or was currently under treatment. They reported $22 \%$ of their sample was positive for $\mathrm{Gl}$ problems, which were primarily constipation and diarrhea (Nikolov et al. 2009).

A few other studies attempted to not only characterize Gl disorders in children with ASD but also make comparisons to other groups of children. Valicenti-McDermott et al. carried out a cross-sectional case-control study ( $\mathrm{n}=50$ in each group) in English and Spanish-speaking families with ASD, TD and DD (Valicenti-McDermott et al. 2006). Children between 1 and 18 years of age were matched for age, gender and ethnicity, and findings indicated a lifetime reported history of 1 or more GI symptoms was higher in cases (ASD $70 \%$ ) than both controls (TDs 28 $\%$, DDs $42 \%)$. Another recent case-control population based study ( $\mathrm{n}=$ 121 cases, $n=242$ controls) in Olmstead County, Minnesota, where >95 $\%$ of medical care is provided by Olmstead Medical Center/Mayo Clinic, medical charts provided data for GI symptoms from birth to 21 years of age (Ibrahim et al. 2009). Out of the five Gl categories compared, only constipation and food selectivity were higher in ASD cases as compared with controls, but potential inclusion of illness episodes in a 21 year period could have skewed results, and $98 \%$ of racial/ethnic makeup was white, raising the issue of generalizability of findings. Finally, in a study using data from the Autism Genetic Resource Exchange (AGRE) where only families with multiple affected members are included, parents reported significantly more Gl problems in children with ASD than in their unaffected siblings ( 42 vs. $12 \%$; Wang et al. 2011). Furthermore, this was the first study to report that having increased autism severity was associated with higher odds of GI problems.

In light of the existing literature of variable methodologies and findings, our study provides the largest ethnically diverse populationbased case-control study to date that compares GI problems for children with clinically confirmed: (1) ASD, (2) DD and (3) TD. It also is the first large population-based sample that examines the relationship of Gl symptoms and maladaptive behaviors. Understanding the magnitude of GI problems and their effects on behavior can provide new insight for more effective and appropriate treatment of children who suffer from these problems. 


\section{Methods}

\section{Study Design and Sample}

The CHildhood Autism Risks from Genetics and the Environment (CHARGE) study is an ongoing population based case-control study with participants sampled from three strata: children with (ASD), children with DD but not ASD, and children selected from the general population (Hertz-Picciotto et al. 2006). Recruitment began in April 2003, and a total of 1,513 participants were enrolled in this CHARGE study sample from April 2003 through May 2011. All participating children meet the following criteria: (a) are between the ages of 24 and 60 months, (b) live with at least one biologic parent, (c) have a parent who speaks English or Spanish, (d) were born in California, and (e) reside in one of the catchment areas of a specified list of Regional Centers in California. All subjects were assessed to confirm diagnostic group and parents completed standardized interviews and questionnaires about their children's past history and present behavior and functioning. Diagnosis of ASD was confirmed in all subjects by the Autism Diagnostic Interview-Revised (ADI-R) (Le Couteur et al. 1993) and the Autism Diagnostic Observation Schedules (ADOS) (Lord et al. 2003). Details have been described previously (Hertz-Picciotto et al. 2006) in which children that met full criteria for autism on both the ADI-R and ADOS were classified as autism (AU), and children that came within two points of meeting criteria for autism on the communications or social domains of the ADI-R and met criteria on ADOS were classified as ASD. Since there was little difference in Gl symptoms between the AU and ASD groups, we have combined them, hereafter designated as ASD for comparisons among children with an ASD, DD and TD. All children were assessed with the Mullen Scales of Early Learning (Mullen 1995) and the Vineland Adaptive Behavior Scales (VABS) (Sparrow 1984). Children recruited into the DD or TD group were also screened with the social communication questionnaire (SCQ); children with scores at or above 15 points were referred for assessment of ASD using the ADOS and ADI-R.). We defined DD based on composite scores for Mullen Scales of Early Learning (MSEL) and VABS. A child was classified as DD if he/she did not meet criteria for ASD, scored $<70$ on either MSEL or VABS and scored $<77$ on the other assessment. Children were classified as TD if their MSEL and 
VABS scores were both $>70$ and they scored $<15$ on the SCQ. For purposes of this analysis, we excluded participants missing the Gastrointestinal History $(\mathrm{GIH})$ questionnaire $(\mathrm{n}=313)$, siblings of target children $(n=26)$, participants with an incomplete or pending diagnosis ( $n=187$ ), those with evidence of developmental delay on only one of two instruments $(n=24)$ and those missing data on child's race ( $n$ =3). The final study population therefore consisted of 960 children.

The study was approved by institutional review boards for the State of California and the University of California, Davis and Los Angeles. Informed consent is obtained for all participants prior to data collection.

\section{Measures and Procedures}

Prior to clinic visits, participants are mailed several self administered questionnaires including the CHARGE GIH and the Aberrant Behavior Checklist $(A B C)$. Maladaptive behavior was measured using five subscales of the $A B C$ : irritability (15 items), lethargy/social withdrawal (16 items), stereotypy (7 items), hyperactivity (16 items) and inappropriate speech (4 items; Aman and Singh 1994). ABC subscales scores ranged based on the number of items scored using a 4 point Likert scale $(0=$ Not at all a problem; $1=$ Problem slight in degree; 2 = Problem moderately serious; 3 = Problem severe in degree). The $\mathrm{GIH}$ includes 10 Likert scale items ( $0=$ never; $1=$ rarely; $2=$ sometimes; 3 = frequently; 4 = always) for each current gastrointestinal symptom (abdominal pain, gaseousness/ bloating, diarrhea, constipation, pain on stooling, vomiting, sensitivity to foods, difficulty swallowing, blood in stool and blood in vomit). "Current" was defined as the past 3 months. Additionally, the GIH includes four (yes/ no) questions asking about the presence of food allergies, diet restrictions, food dislikes and whether any GI diagnosis has ever been given. Finally, there are open-ended questions asking parents to list: food allergies; reasons for diet/food restrictions; and what Gl condition was diagnosed. Other data collected as part of the CHARGE Study protocol include demographics and medications used in the last month. A pediatrician reviewed our list of medications and declared a vast majority of them were known to have some Gl side effects, including medications for cold symptoms, allergies, pain relief, and antibiotics. We did not control for medications known to be used in the treatment of Gl 
symptoms such as anti-diarrheal medications or those used to treat constipation or gastroesophageal reflux disorder (GERD).

\section{Statistical Procedures}

Demographic characteristics were examined across groups for ASD, DD and TD using likelihood ratio Chi square tests for categorical variables. We dichotomized the Likert scale items on the GIH into symptoms that occur with 'high' (items ranked either frequently or always) versus 'low' frequency (items ranked never, rarely or sometimes). We tested for differences in reported frequency of Gl symptoms and food allergies, diet restrictions, strong food dislikes, and GI diagnoses using the likelihood ratio Chi square statistic. Odds ratios adjusted for child age, child gender and maternal education, along with $95 \%$ confidence intervals $(\mathrm{Cl})$, were calculated for a report of high frequency for at least one GI symptom, and then separately for each individual Gl symptom, comparing ASD and DD case groups with TD as the reference. To also control for the possible confounding effect of medications known to have $\mathrm{Gl}$ side effects, an additional set of analyses was conducted on a smaller sample size ( $n=622$, due to missing medication data). Within the ASD and DD groups, we used Wilcoxon rank sum test or t-tests to examine differences in scores on the five subscales of the $A B C$, comparing children with high versus low frequency of Gl symptoms.

Responses provided by parents for open-ended Gl questions were categorized and tallied by the first author (i.e., reported food allergies, reasons for diet restrictions, and reported Gl diagnoses) and categories were reviewed by remaining authors, one of whom is a clinician. All statistical analyses were carried out using SAS version 9.2 (SAS Institute Inc., Cary, NC, USA).

\section{Results}

\section{Study Population Characteristics}

Mothers of children with developmental delay tended to have less formal education than mothers of children with TD or ASD. Roughly half of the study population was white, while approximately one-third 
was of Hispanic origin. Reported medication use was higher in children with DD compared to children with TD or ASD (Table 1).

Table 1 Characteristics and medication use for cases autism spectrum disorder (ASD), developmental delay (DD) and controls typical development (TD). CHARGE study 2003-2011

\begin{tabular}{|c|c|c|c|c|}
\hline & $\begin{array}{l}\text { ASD } \\
n=499\end{array}$ & $\begin{array}{l}\text { Controls TD } \\
\mathrm{n}=324\end{array}$ & $\begin{array}{l}\text { DD } \\
\mathrm{n}=137\end{array}$ & $p$ value $^{a}$ \\
\hline Maternal age & & 0.29 & & \\
\hline Mother's age $\geq 35$ at delivery & $27.1 \%$ & $22.2 \%$ & $25.6 \%$ & \\
\hline Maternal education & & & & $<0.0001$ \\
\hline Less than high school & $3.8 \%$ & $5.3 \%$ & $15.3 \%$ & \\
\hline High school diploma/GED & $9.8 \%$ & $11.1 \%$ & $13.9 \%$ & \\
\hline Some college/2 year degree & $41.7 \%$ & $32.4 \%$ & $40.2 \%$ & \\
\hline Bachelor degree & $28.5 \%$ & $34.6 \%$ & $25.6 \%$ & \\
\hline Graduate or professional degree & $16.2 \%$ & $16.7 \%$ & $5.1 \%$ & \\
\hline Race/ethnicity (child) & & & & 0.02 \\
\hline White & $50.5 \%$ & $52.5 \%$ & $41.6 \%$ & \\
\hline Hispanic & $32.1 \%$ & $29.0 \%$ & $39.4 \%$ & \\
\hline Black & $2.4 \%$ & $1.9 \%$ & $5.1 \%$ & \\
\hline Asian & $5.6 \%$ & $2.5 \% 1$ & $.5 \%$ & \\
\hline Pacific Islander & $0.0 \%$ & $0.3 \%$ & $0.0 \%$ & \\
\hline Multi-racial & $9.4 \%$ & $13.9 \%$ & $12.4 \%$ & \\
\hline Male gender & $85.8 \%$ & $83.0 \%$ & $63.5 \%$ & $<0.0001^{\mathrm{a}}$ \\
\hline Child age in years ${ }^{b}$ & & & & 0.004 \\
\hline 2 year olds (24-35 months) & $22.4 \%$ & $30.6 \%$ & $14.6 \%$ & \\
\hline 3 year olds (36-47 months) & $36.1 \%$ & $36.4 \%$ & $38.0 \%$ & \\
\hline 4 year olds (48-59 months) & $39.1 \%$ & $31.2 \%$ & $46.0 \%$ & \\
\hline 5 year olds (60 months ?) & $2.4 \%$ & $1.9 \%$ & $1.5 \%$ & \\
\hline Regression status (cases only) & $44.9 \%$ & na & na & \\
\hline \multicolumn{5}{|l|}{$\mathrm{MSEL}^{\mathrm{c}}$} \\
\hline Mean \pm SD & $61.5 \pm 17.8$ & $106 \pm 17$ & $453.6 \pm 7.1$ & \\
\hline Range & $49-136$ & $70-149$ & $49-76$ & \\
\hline \multicolumn{5}{|l|}{ VABSd } \\
\hline Mean \pm SD & $64.5 \pm 12.6$ & $104.5 \pm 15$ & $58.9 \pm 8.8$ & \\
\hline Range & $40-150$ & $70-145$ & $33-76$ & \\
\hline Medication use & $\mathrm{n}=465$ & $n=315$ & $n=131$ & \\
\hline \multicolumn{5}{|l|}{ Ever used any } \\
\hline conventional treatments? & $23.2 \%$ & $10.5 \%$ & $39.7 \%$ & $<0.0001$ \\
\hline psychiatric treatments? & $1.5 \%$ & $0.0 \%$ & $3.8 \%$ & 0.002 \\
\hline anticonvulsants or benzodiazepines? & $3.7 \%$ & $0.0 \%$ & $13.7 \%$ & $<0.0001$ \\
\hline CAM treatment? & $26.7 \%$ & $3.5 \%$ & $19.9 \%$ & $<0.0001$ \\
\hline
\end{tabular}

$p$ values calculated for comparisons across groups using Chi-square test or Fisher's exact test

a The TD group was frequency matched to the projected gender distribution of the autism cases, while the DD group was not matched at all

b Reflects age at assessment which occurs within a few months after recruitment. All children were recruited between 24 and 60 months of age

c Mullen Scales of Early Learning

d Vineland Adaptive Behavior Scales 
Table 2 Comparison of 'Current' Gl symptoms (in the past 3 months) for cases (ASD) and controls (TD and DD). CHARGE study 2003-2011

$\begin{array}{rrrlrrr}\text { ASD } & \text { TD } & \text { DD } & p \text { value } & \text { AU } & \text { ASD } & p \text { value }^{\mathrm{b}} \\ \mathrm{n}=499 & \mathrm{n}=324 & \mathrm{n}=137 & & \mathrm{n}=339 & \mathrm{n}=160 & \\ (\%) & (\%) & (\%) & (\%) & (\%) & \end{array}$

\begin{tabular}{lrrrrrrr}
\hline Gl symptoms $^{b}$ & & & & & & & \\
$\quad$ Abdominal pain & 5.1 & 1.6 & 3.9 & 0.03 & 5.9 & 3.5 & 0.27 \\
Gaseousness/bloating sensation & 11.0 & 2.0 & 2.3 & $<0.0001$ & 11.7 & 9.6 & 0.51 \\
Diarrhea & 13.0 & 1.6 & 6.1 & $<0.0001$ & 16.1 & 6.4 & 0.002 \\
Constipation & 15.5 & 3.5 & 15.8 & $<0.0001$ & 16.8 & 12.5 & 0.22 \\
Pain on stooling & 6.2 & 1.6 & 5.5 & 0.004 & 7.4 & 3.4 & 0.08 \\
Vomiting & 2.9 & 0.3 & 6.2 & 0.0006 & 2.7 & 3.2 & 0.76 \\
Sensitivity to foods & 31.0 & 4.5 & 11.1 & $<0.0001$ & 32.1 & 28.9 & 0.49 \\
Difficulty swallowing & 4.2 & 0.3 & 4.6 & 0.0005 & 4.0 & 4.6 & 0.77 \\
Blood in stools & 0.4 & 0.3 & 0.8 & 0.62 & 0.6 & 0.0 & 1 \\
Blood in vomit & 0.2 & 0.0 & 0.0 & 1 & 0.0 & 0.6 & 0.32 \\
Presence of related issues & & & & & & & \\
Food allergies Y/N & 23.0 & 11.1 & 13.5 & $<0.0001$ & 19.7 & 20.3 & 0.88 \\
Food restrictions Y/N & 37.4 & 10.4 & 21.6 & $<0.0001$ & 40.2 & 31.4 & 0.06 \\
Food dislikes Y/N & 63.5 & 34.0 & 34.6 & $<0.0001$ & 63.8 & 62.9 & 0.89 \\
Gl diagnosis Y/N & 7.5 & 1.9 & 22.9 & $<0.0001$ & 7.8 & 6.9 & 0.7 \\
\hline
\end{tabular}

Those reporting symptoms occur 'frequently' or 'always' on Likert scale

a $p$ values calculated for comparisons across groups using Chi-square test or Fisher's exact test

${ }^{b}$ Number of missing data for entire sample for the first ten Gl question varies from 29 to 77

c Number of missing data for entire sample for the last four questions varies from 19 to 38 (except 'food dislikes' missing = 332)

\section{Comparisons of GI Symptom Reports Across Diagnostic Groups of ASD, DD and TD}

Parent report of at least one frequent Gl symptom was significantly higher for children with ASD and children with DD, compared to children with TD. Comparison of parent reports suggests that children with either ASD or DD are far more likely to have frequent constipation, diarrhea and difficulty swallowing than children with TD (Table 2). Group differences in GI symptoms for children with ASD held up after adjusting for child age, child gender, and maternal education (Table 3). Children with ASD were at least three times more likely to experience frequent Gl symptoms than children with TD: abdominal pain, pain on stooling, constipation, gaseousness/bloating, diarrhea, sensitivity to foods, as well as vomiting and difficulty swallowing, which were very rare in TD controls. Similarly, reported food allergies, food restrictions, and food dislikes were highest in children with ASD (Table 
Table 3 Odds ratio of 'Current' GI symptoms (in the past 3 months) for ASD with typical development (TD) as reference group. CHARGE study 2003-2011

\begin{tabular}{|c|c|c|c|c|c|c|}
\hline Gl symptoms & $\begin{array}{l}\text { Unadjusted } \\
\text { odds ratio }\end{array}$ & $95 \% \mathrm{Cl}$ & $\begin{array}{l}\text { Adjusted } \\
\text { odds ratio }\end{array}$ & $95 \% \mathrm{Cl}$ & $\begin{array}{l}\text { Adjusted } \\
\text { odds ratiob }\end{array}$ & $95 \% \mathrm{Cl}$ \\
\hline \multicolumn{7}{|l|}{$A S D$ versus $T D$} \\
\hline \multicolumn{7}{|c|}{ Full study populationc $(\mathrm{n}=960)$} \\
\hline Abdominal pain & 3.28 & $(1.23-8.72)$ & 3.26 & $(1.21-8.78)$ & na & na \\
\hline Gaseousness/bloating & 6.15 & $(2.60-14.54)$ & 6.43 & $(2.71-15.29)$ & na & na \\
\hline Diarrhea & 9.08 & $(3.61-22.84)$ & 9.43 & $(3.73-23.83)$ & na & na \\
\hline Constipation & 5.02 & $(2.62-9.62)$ & 5.41 & $(2.81-10.41)$ & na & na \\
\hline Pain on stooling & 4.03 & (1.54-10.52) & 4.20 & $(1.60-11.03)$ & na & na \\
\hline Vomiting & 9.08 & $(1.19-69.38)^{\mathrm{e}}$ & 9.83 & $(1.28-75.40)^{\mathrm{e}}$ & na & na \\
\hline Sensitivity to foods & 9.51 & $(5.37-16.83)$ & 9.67 & $(5.44-17.18)$ & na & na \\
\hline Difficulty swallowing & 13.29 & $(1.77-99.53)^{\mathrm{e}}$ & 12.57 & $(1.67-94.71)$ & na & na \\
\hline \multicolumn{7}{|c|}{ Subset with medication datad $(n=622)$} \\
\hline Abdominal pain & 3.14 & $(1.11-8.87)$ & 3.01 & $(1.05-8.64)$ & 2.85 & $(0.99-8.24)$ \\
\hline Gaseousness/bloating & 6.13 & $(2.31-16.25)$ & 6.14 & $(2.29-16.49)$ & 5.93 & $(2.20-15.98)$ \\
\hline Diarrhea & 6.35 & $(2.16-18.65)$ & 6.09 & $(2.06-18.03)$ & 6.19 & (2.09-18.33) \\
\hline Constipation & 5.76 & $(2.63-12.62)$ & 6.07 & $(2.74-13.43)$ & 6.06 & (2.74-13.42) \\
\hline Pain on stooling & 2.8 & $(0.98-7.97)$ & 2.91 & $(1.01-8.41)$ & 2.84 & $(0.98-8.21)$ \\
\hline Vomiting & 7.21 & $(0.88-59)^{e}$ & 7.72 & $(0.93-64.16)^{\mathrm{e}}$ & 8.04 & $(0.97-67)^{\mathrm{e}}$ \\
\hline Sensitivity to foods & 8.61 & $(4.62-16.07)$ & 8.6 & $(4.58-16.14)$ & 8.57 & (4.56-16.09) \\
\hline Difficulty swallowing & 7.23 & $(0.88-59.20)^{e}$ & 6.56 & $(0.79-54.3)^{\mathrm{e}}$ & 6.89 & $(0.83-57.5)^{\mathrm{e}}$ \\
\hline
\end{tabular}

Those reporting symptoms occur 'frequently' or 'always' on Likert scale

${ }^{a}$ Adjusted for child's age, gender, maternal education

${ }^{\mathrm{b}}$ Adjusted for child's age, child's gender, maternal education, and medication

'TD group $n=324$, ASD group $n=499$, DD group $n=137$

d TD group $n=257$, ASD group $n=254$, DD group $n=111$

e Wide confidence interval $(\mathrm{Cl})$ is a result of only one control having this symptom

2). However, parent report of a Gl diagnosis was highest in children with DD compared to children with ASD and TD. Moreover, children with DD were at least three times more likely than children with TD to experience frequent sensitivity to foods, pain on stooling, diarrhea, constipation, as well as difficulty swallowing and vomiting.

Analyses using those with parent-report medication data confirm that after adjusting for medications with potential GI side effects, most findings were essentially unchanged: children with ASD are approximately six to eight times more likely to report frequent gaseousness/ bloating, constipation, diarrhea and sensitivity to foods regardless of which variables were controlled (Table 3). Similarly, parents of children with DD were five-fold more likely to report constipation, as well as significantly more likely to report difficulty swallowing and vomiting compared to parents of TD children after adjusting for medications 
Table 4 Odds ratio of 'Current' GI symptoms (in the past 3 months) for DD with TD as reference group. CHARGE study 2003-2011

\begin{tabular}{|c|c|c|c|c|c|c|}
\hline Gl symptoms & $\begin{array}{l}\text { Unadjusted } \\
\text { odds ratio }\end{array}$ & $95 \% \mathrm{Cl}$ & $\begin{array}{l}\text { Adjusted } \\
\text { odds ratioa }\end{array}$ & $95 \% \mathrm{Cl}$ & $\begin{array}{l}\text { Adjusted } \\
\text { odds ratio }\end{array}$ & $95 \% \mathrm{Cl}$ \\
\hline \multicolumn{7}{|l|}{$D D$ versus $T D$} \\
\hline \multicolumn{7}{|c|}{ Full study population ${ }^{c}(\mathrm{n}=960)$} \\
\hline Abdominal pain & 2.48 & $(0.71-8.73)$ & 2.09 & $(0.56-7.73)$ & na & na \\
\hline Gaseousness/bloating & 1.19 & $(0.29-4.86)$ & 1.39 & $(0.33-5.76)$ & na & na \\
\hline Diarrhea & 3.97 & $(1.27-12.36)$ & 4.71 & $(1.48-15.01)$ & na & na \\
\hline Constipation & 5.15 & $(2.41-11.02)$ & 4.77 & $(2.17-10.46)$ & na & na \\
\hline Pain on stooling & 3.58 & $(1.12-11.50)$ & 3.70 & $(1.11-12.31)$ & na & na \\
\hline Vomiting & 20.2 & $(2.5-163.19)^{\mathrm{e}}$ & 24.00 & $(2.88-200.24)^{\mathrm{e}}$ & na & na \\
\hline Sensitivity to foods & 2.64 & $(1.22-5.72)$ & 3.14 & $(1.43-6.92)$ & na & na \\
\hline Difficulty swallowing & 14.85 & $(1.77-124.65)^{\mathrm{e}}$ & 10.42 & $(1.20-90.27)^{\mathrm{e}}$ & na & na \\
\hline \multicolumn{7}{|c|}{ Subset with medication data $(n=622)$} \\
\hline Abdominal pain & 1.46 & $(0.34-6.24)$ & 1.33 & $(0.30-6.01)$ & 1.19 & $(0.23-5.45)$ \\
\hline Gaseousness/bloating & 0.95 & $(0.18-4.99)$ & 1.08 & $(0.20-5.85)$ & 0.93 & $(0.17-5.10)$ \\
\hline Diarrhea & 2.41 & $(0.59-9.80)$ & 2.38 & $(0.56-10.05)$ & 2.51 & $(0.59-10.64)$ \\
\hline Constipation & 5.28 & $(2.18-12.75)$ & 4.92 & $(1.97-12.32)$ & 4.91 & $(1.96-12.31)$ \\
\hline Pain on stooling & 2.53 & $(0.72-8.94)$ & 3.06 & $(0.83-11.33)$ & 2.9 & $(0.78-10.83)$ \\
\hline Vomiting & 15 & $(1.78-126.21)^{e}$ & 17.83 & $(2.01-158.34)^{e}$ & 20.86 & $(2.3-189.5)^{\mathrm{e}}$ \\
\hline Sensitivity to foods & 1.75 & $(0.73-4.24)$ & 2.03 & $(0.82-5.04)$ & 2.01 & $(0.81-5)$ \\
\hline Difficulty swallowing & 12.32 & $(1.42-106.69)^{e}$ & 9.36 & $(1.02-85.77)^{\mathrm{e}}$ & 12.32 & $(1.31-115.83)^{\mathrm{e}}$ \\
\hline
\end{tabular}

Those reporting symptoms occur 'frequently' or 'always' on Likert scale

${ }^{a}$ Adjusted for child's age, gender, maternal education

${ }^{\mathrm{b}}$ Adjusted for child's age, child's gender, maternal education, and medication

${ }^{c}$ TD group $n=324$, ASD group $n=499$, DD group $n=137$

d TD group $n=257$, ASD group $n=254$, DD group $n=111$

e Wide confidence interval $(\mathrm{Cl})$ is a result of only one control having this symptom

(Table 4). The exclusion of those without medication data appears to have resulted in a somewhat lower prevalence of reported diarrhea.

We did not find any meaningful differences between ASD children with DD ( $n=114)$, ASD without DD $(n=377)$ and DD without ASD (our DD group), beyond what the previous analyses have already described. Specifically, Gl symptoms did not significantly differ comparing ASD with DD versus ASD without DD, with the exception of vomiting. Vomiting is a relatively infrequent $\mathrm{Gl}$ symptom reported, and ASD without DD had higher occurrence than ASD with DD ( $p=0.02$; Table 1S). 


\section{Comparisons by Severity of Autism Spectrum Disorder}

Comparisons of $A U$ versus ASD, as originally defined in the CHARGE study, suggest that $\mathrm{Gl}$ symptoms and related issues are similar between the two groups, with the exception of diarrhea (Table 2). Children with $A U$ have higher reports of diarrhea compared to children with ASD.

\section{Examination of GI Symptoms and ABC Scores}

We examined the association between GI symptoms and maladaptive behavior scores for the five subscales of the $A B C$. In children with ASD, four out of five behavior subscales (irritability, social withdrawal, stereotypy and hyperactivity) on the $A B C$ were significantly higher in children with frequent occurrences of abdominal pain, gaseousness, diarrhea and constipation as compared to children with no frequent Gl symptoms (Table 5). In addition, two or three of the behavior subscales (irritability, social withdrawal, and stereotypy) on the $A B C$ were significantly higher in children with other frequent Gl symptoms (pain on stooling, sensitivity to food, and difficulty swallowing). For children with $D D$, maladaptive behavior scores for the $A B C$ yielded a pattern of associations only for diarrhea: scores were higher for irritability (GI positive 16.7 SE 4.6, GI negative 8.6 SE 0.9, $p=0.06$ ), social withdrawal (GI positive 8.6 SE 2.6, GI negative 4.0 SE 0.5, $p=0.09$ ) and hyperactivity subscales (GI positive 22.1 SE 4.8, GI negative 11.0 SE 1.0, $p=$ 0.02 ) in children whose parent reported frequent diarrhea versus infrequent diarrhea (Table 2S).

\section{Open-Ended questions}

Descriptive analyses of parental responses to related open ended GI questions indicated the most frequently reported food sensitivity or allergy for all diagnostic groups was dairy/casein (14.6\% ASD, 6.6 $\%$ DD and $5.3 \%$ TD). For children with ASD, other commonly reported food allergies or sensitivities included the grains category (including gluten, $7.8 \%$ ) and nuts/soy (7\%). The most commonly cited reason for food restrictions in children with ASD was child selectivity (8.6\%) followed by adverse Gl symptoms (8.2\%); and for children with DD 


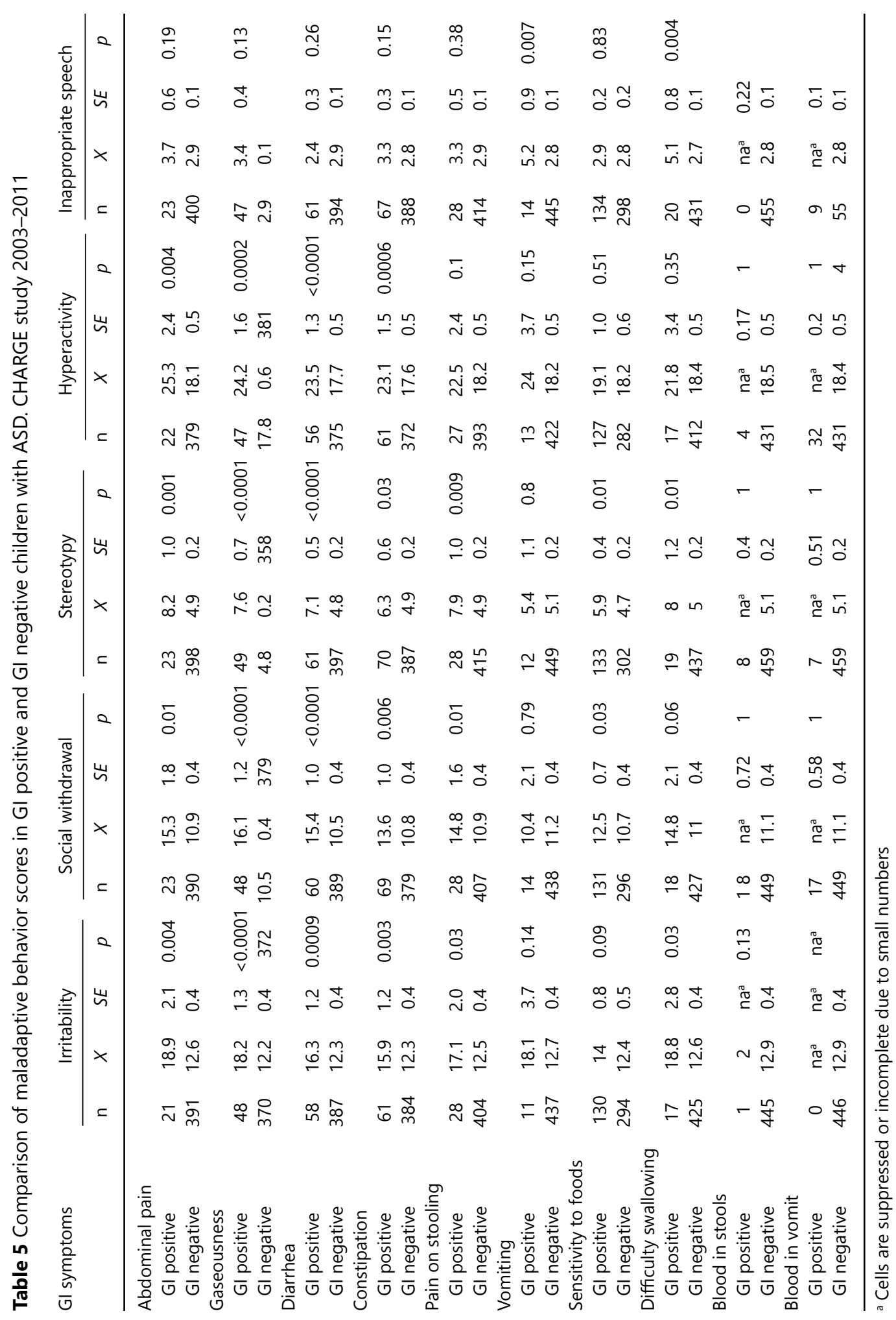


and TD it was adverse Gl symptoms (DD $10.2 \%$, TD $4 \%$ ). The most commonly reported $\mathrm{Gl}$ diagnosis across all groups was gastroesophageal reflux disorder (GERD), although in children with ASD $(n=18)$, half of these were reported to have resolved. Only 2 cases were reported in the disease category for celiac disease/colitis, 1 in ASD (0.4 $\%$ ) and 1 in DD (1.5 \%), whereas an additional 6 cases in ASD (1.2\%) reported malabsorption, leaky gut or abnormal stool and five children with DD (nearly $4 \%$ ) had Gl anatomical abnormalities. Five cases of dysbiosis (yeast in stool) were also reported in children with ASD (1\%).

We also examined the types of combinations for children with two or more reported frequent $\mathrm{Gl}$ symptoms in the last 3 months. In a majority of cases, diarrhea and constipation occur in mutually exclusive groups. For example, in children with ASD, only 9 reported both diarrhea and constipation, while 29 reported diarrhea in combination with other symptoms, and 44 reported constipation with other symptoms. In children with DD, only 3 children had both diarrhea and constipation, while another 3 reported diarrhea in combination with other $\mathrm{Gl}$ symptoms, and 8 reported constipation in combination with other $\mathrm{Gl}$ symptoms.

\section{Discussion}

Our findings from the largest population-based case-control study of GI problems in children with ASD and DD confirmed using standardized tests indicate that these symptoms, particularly constipation and diarrhea, affect children with ASD and DD far more often than children with TD. Compared to children with TD, children with ASD and DD were at least three times more likely to experience a higher frequency of most $\mathrm{Gl}$ symptoms. After adjustment for medications, the odds ratios were changed very little (with the exception of difficulty swallowing in the ASD analysis, reported for only one TD control). Exceedingly strong differences in reported frequency of diarrhea, constipation, gaseousness/bloating and sensitivity to foods were robust across models, with prevalences five to nine-fold higher in ASD. What remains to be understood are the mechanisms or contributing factors that can help explain these differences.

Comparison of our findings to other studies yielded some notable similarities. For example, Valicenti-McDermott et al. in a New York 
sample of slightly older children (mean age 7.6 years, SD \pm 3.6 ) reported food selectivity at similar prevalences (ASD $60 \%$, TD $22 \%$, and DD $36 \%$ ) as food dislikes in our California sample (ASD 63.5\%, TD $34 \%$, and DD $34.6 \%$ ). Abnormal stool patterns (defined as recurrent passage of $\geq 3$ large unformed stools daily for $>4$ weeks) were reported only slightly more often in cases (ASD $18 \%$, TD $4 \%$, DD 2 $\%)$; as compared with reports of diarrhea in our sample (ASD $13 \%$, TD $2 \%$, DD $6 \%$ ).

In contrast to our findings, Ibrahim et al. found that the overall incidence of Gl symptoms did not differ between children with autism and gender- and age-matched children with typical development. In the Ibrahim study, information from medical charts covered birth to 21 years of age: constipation; diarrhea; abdominal bloating, discomfort or irritability; gastroesophageal reflux or vomiting; and feeding issues or food selectivity (Ibrahim et al. 2009). However, it was not indicated whether these findings included illnesses such as the flu, which could account for null findings, especially since their study investigated cumulative incidence of $\mathrm{GI}$ problems over a long time period. In the CHARGE study, we collected information on GI problems occurring in the last 3 months.

Interestingly, food selectivity for children with ASD is a common thread in several studies (Valicenti-McDermott et al. 2006; Ibrahim et al. 2009). Ibrahim et al. concluded that constipation and food selectivity were attributed to behavioral characteristics of children with ASD such as ritualistic tendencies, need for routine, and insistence on sameness, rather than being indicative of GI pathology. Our results provide some support for these contentions in that children with ASD had not only the highest reports of food dislikes (a proxy for food selectivity), but also the highest odds of reporting $\mathrm{Gl}$ symptoms not explained by other factors such as the child's age, medication or maternal education. It is plausible that a child with notable food dislikes or selectivity for a variety of reasons such as taste, texture, or temperature, might consume a relatively self-restricting diet that could reduce the variety of foods and nutrients needed to maintain healthy gut function. Only one study to date has examined dietary habits as a possible link between gastrointestinal dysfunction and autism, and findings suggested there was no association (Gorrindo et al. 2012).

To our knowledge, only the Valicenti-McDermott study and ours offer a comprehensive comparison of GI problems in ASD children 
versus those having other developmental disorders. One recent study assessed the prevalence of various medical conditions, including one Gl symptom, using a nationally representative sample of children ages 3-17 years and found the prevalence of frequent diarrhea/ colitis in the past 12 months was seven times more likely in children with autism [6.8 \%, aOR 7.1, $95 \% \mathrm{Cl}$ (3.9-12.8)] and three times more likely in children with a developmental disability [3 \%, aOR 3.5, $95 \% \mathrm{Cl}$ (2.7-4.5)] than children with typical development (0.9\%; Schieve et al. 2012).

In comparing our findings with others, we note that despite the similarities in the DD groups between Valicenti- McDermott and our study, the two groups are not quite the same. Our CHARGE Study DD group is designated as having a developmental delay, regardless of the cause, i.e., it includes those with known chromosomal, genetic or mitochondrial disorders whereas Valicenti- McDermott designates the DD group as children with developmental disabilities and they exclude from both the ASD and DD groups, those with "known genetic" syndromes or disorders. In either case, comparisons to children with DD provide information on the specificity of findings in ASD: overall, GI symptoms were more prevalent in both ASD and DD children when comparing with TD children, signifying a lack of specificity in regard to this phenotypic presentation. When we compared subsets within the ASD group, ASD children with DD versus ASD children without DD did not, in the main, differ in their Gl symptoms. The one exception was for vomiting, but given that no other symptoms differed and that it was a relatively infrequently reported $\mathrm{Gl}$ symptom, the finding is likely due to chance.

Our findings in children with ASD demonstrating a consistent relationship between $\mathrm{Gl}$ symptoms and maladaptive behaviors have perhaps special clinical translational significance, with possible implications for treatment approaches. Similar to the strong and significant relationship we found between $\mathrm{Gl}$ symptoms and increased measures of irritability, social withdrawal, stereotypy, and hyperactivity, Nikolov et al. observed that children with PDDs and GI problems (all symptoms combined) showed greater symptom severity on measures of irritability and social withdrawal on the $A B C$, as well as anxiety, and to our knowledge is the only other study addressing $\mathrm{Gl}$ problems and behavior of children with ASDs (Nikolov et al. 2009). It is plausible that a chronic GI symptom, which can cause pain, discomfort 
and anxiety, could contribute to increased irritability and social withdrawal, particularly in someone with deficits in social and communicative skills. Furthermore, for a child with ASD, increased stereotypy and hyperactivity may represent coping mechanisms for an uncomfortable and unpredictable GI condition. A recent report found that behavioral characteristics hypothesized to be expressions of GI problems are common in children with ASD, yet not specific to those with Gl problems (Maenner et al. 2012). Therefore the authors suggested that the presence of these behaviors would not be useful on their own for screening or identifying children requiring $\mathrm{Gl}$ evaluation. However, many ASD children, most especially those who are non-verbal, often do not present with symptoms typically recognized by many primary care physicians or specialists as being GI related, such as self-injurious behavior (SIB) and aggression that may be responses to pain and/ or discomfort. Until clinicians and therapists consider a thorough $\mathrm{Gl}$ history as a possible explanation for adverse behaviors, Gl disorders in this population will continue to be over-looked and insufficiently treated. Appropriate treatment of Gl symptoms may help alleviate at least some problematic behaviors and improve the quality of life in children with ASD along with their families. A recent consensus report on the evaluation, diagnosis and treatment of $\mathrm{Gl}$ disorders in children with ASD supports the use of medical investigation for problem behaviors (Statement 7) and provides examples and guidance for clinicians (Buie et al. 2010a).

In children with DD, diarrhea was the only GI symptom associated with measures of maladaptive behaviors, specifically irritability, social withdrawal and hyperactivity. As in ASD, clinicians attending to children with DD should inquire about Gl conditions, particularly where behavioral problems or recent changes in behavior have been noted. Larger sample sizes and analyses focused on both idiopathic and specific etiologies of DD should be investigated to understand the role of Gl problems in children with DD, and their relationship with a child's behavior.

Comparisons between children with AU and those with ASD yielded very similar GI profiles with the exception of diarrhea. The ten percentage point higher prevalence in those with the more severe diagnosis $(A U)$ might indicate that greater severity induces more co-morbidity or alternatively, that the presence of chronic diarrhea increases symptom severity. Wang and colleagues also reported on autism symptom 
severity and odds of having Gl problems showing that having "Full Autism" (aOR 14.28, 95 \% Cl 6.22-32.77) or "Almost Autism" (aOR 5.16, $95 \% \mathrm{Cl} 2.02-13.21$ ) were strongly associated with experiencing $\mathrm{Gl}$ problems in multiplex families affected with autism compared to unaffected siblings (Wang et al. 2011). Diarrhea was one of the two most reported GI problems in children with ASD.

Dairy and/or casein were cited most often as food allergens among all groups of children. However, it is unclear to what degree these parent-reports reflect true food allergies versus food sensitivities. At least one group of researchers has demonstrated that in children with ASD, it is nonallergic food hypersensitivity to cow's milk protein (CMP), and not casein that plays a role in Gl symptoms observed in some children with ASD (Jyonouchi et al. 2005). Food allergies are often difficult to diagnose because most intestinal food allergies are cell mediated rather than by immunoglobulin E (IgE) (Buie et al. 2010b). Cell-mediated immunity plays a role in non-allergic food hypersensitivity and reactions take place several hours and even 1-2 days after the intake of the culprit (Jyonouchi et al. 2005). Finally, it is possible that reported food allergens may be neither an allergy or sensitivity, but rather a parent's or child's perception or opinion of a particular food(s).

For children with ASD, parents report child selectivity as the primary reason for diet restriction, followed closely by adverse GI symptoms. Although it makes sense for child refusal and adverse Gl symptoms to be common reasons for diet restrictions, we would have expected more parents to report food allergy as a reason for imposing a diet restriction considering the number of food allergies or sensitivities reported. Diagnoses of celiac disease were rare, which is interesting given the sizable number reporting a food allergy to grains and/ or gluten. A limitation of our study is that these findings are based solely on parent-report rather than medical chart review for diagnostic testing.

Among our study's other limitations is the lack of standardized definitions for $\mathrm{Gl}$ symptoms. This makes it difficult to make comparisons across studies. Our data was based on parent report, so there will be a level of inherent subjectivity, which on the one hand is a limitation, but for children in this age range, parents are likely best suited to follow and report bowel habits and GI symptoms that may not be reliably recorded in medical records. Furthermore, dietary intake information for the children is also lacking. Diarrhea and constipation are 
frequently cited in studies examining Gl symptoms in children with autism, but to date, only one has examined the role of diet in GI problems (Gorrindo et al. 2012). To investigate whether diet plays a role in the prevalence of Gl problems, future studies should attempt to measure diet quality and quantity of key macronutrients with special attention to fiber, water, and fats, in addition to micronutrients such as iron, zinc, vitamin A, vitamin B12, folate and iodine. One small study suggests that children with ASDs may have inadequate intakes of fiber, calcium, iron, vitamin E and vitamin D (Herndon et al. 2009).

Among the strengths of this analysis are: the CHARGE Study population-based sample, which provided a broader ethnic diversity of cases and controls than previous studies; the large sample size of nearly 1,000 children; and adjustment for confounding from both sociodemographic factors and medications. In addition to demonstrating a much higher incidence of parent-reported frequent diarrhea, gaseousness/bloating, constipation, and food sensitivities in 2-5 years old children with ASD versus their typically developing counterparts, this study identified a strong correlation between deviant behaviors and the presence of Gl symptoms in both children with ASD and DD. This underscores the need for clinical medical intervention and attention to $\mathrm{Gl}$ symptoms in these children. Future work examining GI problems in children with ASD and DD should standardize definitions for GI symptoms and more studies need to incorporate measures of dietary intake and nutritional status.

The variety of $\mathrm{Gl}$ symptoms evident within a significant portion of the ASD population may be reflective of the heterogeneity of the disorder in general. Further, the neurotransmitter systems that are active in the brain can also be found to function in the gut, which has been referred to as 'the second brain' (de Theije et al. 2011). Thus, it is possible that research into $\mathrm{Gl}$ symptoms might elucidate aspects of the underlying neurobiological mechanisms associated with the disorder. Investigating the dual role of neurotransmitters active in both the gut and the brain in future studies may advance our understanding of underlying mechanisms important to both.

Acknowledgments - The authors would like to thank Bill Elms and Lora Delwiche for their guidance and support with data management, Melissa Rose and the CHARGE research team. And a special thanks to all the families who took part 
in this research. There are no conflicts of interest to declare by any of the authors. Sponsors of this research included: National Institutes of Health, National Institute of Environmental Health Sciences; Grant numbers: 1 P01 ES11269, 2 P01 ES11269, 1 R01 ES015359, and 3 R01 ES015359-03S2; the US Environmental Protection Agency through the Science to Achieve Results (STAR) program; Grant number: R833292 and R829388; the Medical Investigations of Neurodevelopmental Disorders (M.I.N.D.) Institute; Grant Number: N/A; Autism Speaks; Grant Number: AS 7567.

\section{References}

Aman, M. G., \& Singh, N. N. (1994). Aberrant behavior checklist community. East Aurora, NY: Supplementary Manual.

Buie, T., Campbell, D. B., Fuchs, G. J, 3rd, Furuta, G. T., Levy, J., Vandewater, J., et al. (2010a). Evaluation, diagnosis, and treatment of gastrointestinal disorders in individuals with ASDs: a consensus report. Pediatrics, 125(Suppl 1), S1-S18.

Buie, T., Fuchs, G. J, 3rd, Furuta, G. T., Furuta, G. T., Kooros, K., Levy, J., et al. (2010b). Recommendations for evaluation and treatment of common gastrointestinal problems in children with ASDs. Pediatrics, 125(Suppl 1), S19-S29.

De Theije, C. G., Wu, J., da Silva, S. L., Kamphuis, P. J., Garssen, J., Korte, S. M., et al. (2011). Pathways underlying the gut-to-brain connection in autism spectrum disorders as future targets for disease management. European Journal of Pharmacology, 666(S1), S70-s80. doi:10.1016/j.ejphar.2011.07.013.

Gorrindo, P., Williams, K. C., Lee, E. B., Walker, L. S., McGrew, S. G., \& Levitt, P. (2012). Gastrointestinal dysfunction in autism: parental report, clinical evaluation, and associated factors. Autism Research, 5(2), 101-108.

Herndon, A. C., DiGuiseppi, C., Johnson, S. L., Leiferman, J., \& Reynolds, A. (2009). Does nutritional intake differ between children with autism spectrum disorders and children with typical development? Journal of Autism and Developmental Disorders, 39(2), 212-222.

Hertz-Picciotto I, Croen L, Hansen R, Jones C, Pessah IN (2006). The CHARGE study: an epidemiologic investigation of genetic and environmental factors contributing to autism. Environmental Health Perspectives, 114(7), 1119-1125. http://www.ncbi.nlm.nih.gov/pmc/articles/PMC1513329/pdf/ehp0114-001119. pdf.

Ibrahim, S. H., Voigt, R. G., Katusic, S. K., Weaver, A. L., \& Barbaresi, W. J. (2009). Incidence of gastrointestinal symptoms in children with autism: a populationbased study. Pediatrics, 124(2), 680-686.

Jyonouchi, H., Geng, L., Ruby, A., Reddy, C., \& Zimmerman-Bier, B. (2005). Evaluation of an association between gastrointestinal symptoms and cytokine production against common dietary proteins in children with autism spectrum disorders. Journal of Pediatrics, 146(5), 605-610.

Le Couteur A, Lord C, Rutter M. (1993). The Autism Diagnostic Interview, Revised (ADI-R), Los Angeles, CA. 
Lord C, Rutter M, diLavore PC, Risi S. (2003). Autism Diagnostic Observation Schedule Manual, Los Angeles, CA.

Maenner, M. J., Arneson, C. L., Levy, S. E., Kirby, R. S., Nicholas, J. S., \& Durkin, M. S. (2012). Brief Report: Association between behavioral features and gastrointestinal problems among children with autism spectrum disorder. Journal of Autism and Developmental Disorders, 42, 1520-1525.

Molloy, C. A., \& Manning-Courtney, P. (2003). Prevalence of chronic gastrointestinal symptoms in children with autism and autistic spectrum disorders. Autism, 7, 165-171.

Mullen, E. M. (1995). Mullen scales of early learning. Circle Pines, MN: American Guidance Services.

Nikolov, R. N., Bearss, K. E., Lettinga, J., Erickson, C., Rodowski, M., Aman, M. G., et al. (2009). Gastrointestinal symptoms in a sample of children with pervasive developmental disorders. Journal of Autism and Developmental Disorders, 39(3), 405-413.

Schieve, L. A., Gonzalez, V., Boulet, S. L., Visser, S. N., Rice, C. E., Braun, K. V., et al. (2012). Concurrent medical conditions and health care use and needs among children with learning and behavioral developmental disabilities, National Health Interview Survey, 2006-2010. Research in Developmental Disabilities, 33(2), 467-476.

Sparrow, S. S. (1984). Vineland adaptive behavior scales survey form manual. Circle Pines, MN: American Guidelines Service.

Valicenti-McDermott, M., McVicar, K., Rapin, I., Wershil, B. K., Cohen, H., \& Shinnar, S. (2006). Frequency of gastrointestinal symptoms in children with autistic spectrum disorders and association with family history of autoimmune disease. Journal of Developmental and Behavioral Pediatrics, 27(2 Suppl), S128-S136.

Wang, L. W., Tancredi, D. J., \& Thomas, D. W. (2011). The prevalence of gastrointestinal problems in children across the United States with autism spectrum disorders from families with multiple affected members. Journal of Developmental and Behavioral Pediatrics, 32(5), 351-360. 
Table 1S. Comparison of 'Current' Gl symptomsa (in the past 3 months) for cases (ASD, with \& without DD) and controls (DD). CHARGE study 2003-2011.

\begin{tabular}{lcccc} 
& ASD & & \\
& without & ASD & & \\
& DD & with DD & DD & $P$ value $^{\text {b }}$ \\
& $\mathrm{n}=377$ & $\mathrm{n}=114$ & $\mathrm{n}=136$ & \\
Gl symptoms & $\%$ & $\%$ & $\%$ & \\
Abdominal pain & $5.8 \%$ & $4.7 \%$ & $4 \%$ & 0.82 \\
Gaseousness/bloating sensation & $9.4 \%$ & $11.4 \%$ & $2.4 \%$ & 0.003 \\
Diarrhea & $8.9 \%$ & $14.4 \%$ & $6.2 \%$ & 0.02 \\
Constipation & $13.6 \%$ & $15.5 \%$ & $15.9 \%$ & 0.86 \\
Pain on stooling & $6.5 \%$ & $5.6 \%$ & $5.6 \%$ & 0.94 \\
Vomiting & $5.4 \%$ & $1.6 \%$ & $6.2 \%$ & 0.02 \\
Sensitivity to foods & $29.4 \%$ & $31.6 \%$ & $11.2 \%$ & $<0.0001$ \\
Difficulty swallowing & $5.5 \%$ & $3.3 \%$ & $4.7 \%$ & 0.55 \\
Blood in stools & $0.9 \%$ & $0.3 \%$ & $0.8 \%$ & 0.35 \\
Blood in vomit & $0.9 \%$ & $0 \%$ & $0 \%$ & 0.19 \\
& \multicolumn{4}{l}{} \\
aThose reporting symptoms occur 'frequently' or 'always' on Likert scale \\
bP values calculated using chi-square test; Fisher's exact test used where applicable \\
cNumber of missing data for entire sample varies from 1-39
\end{tabular}




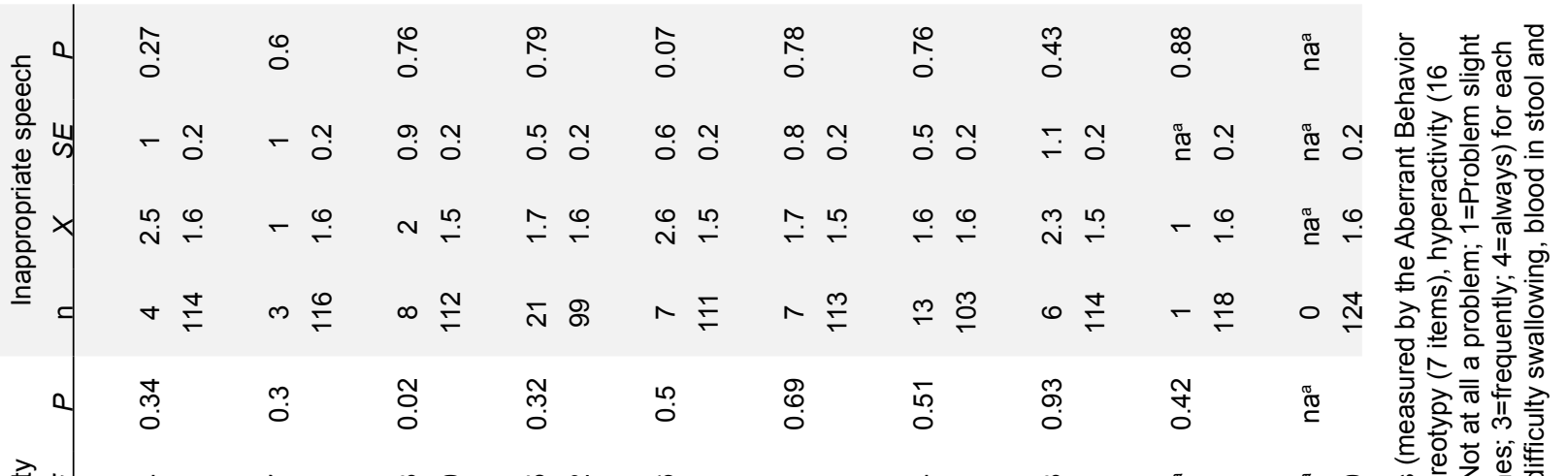

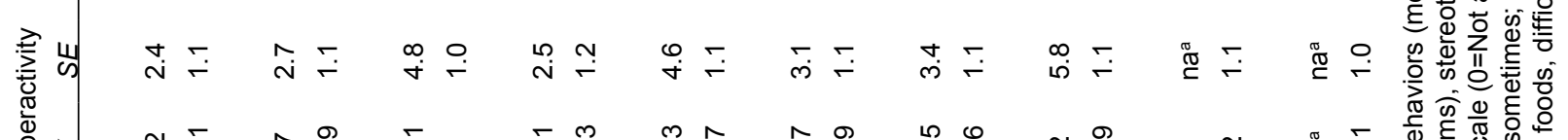

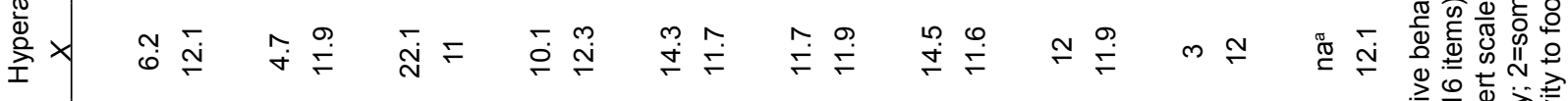

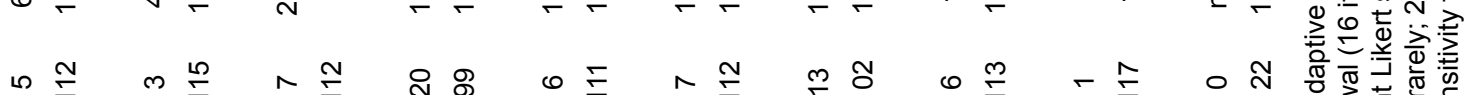

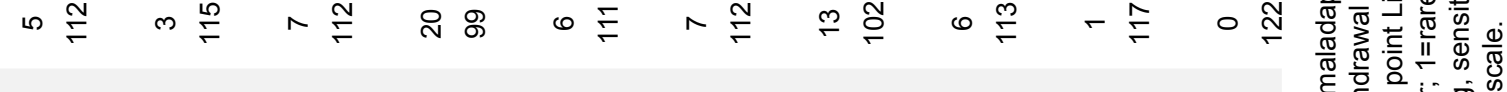

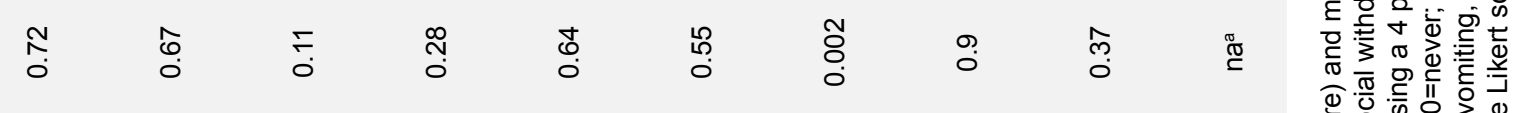

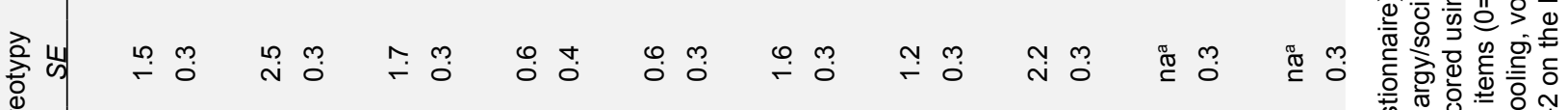

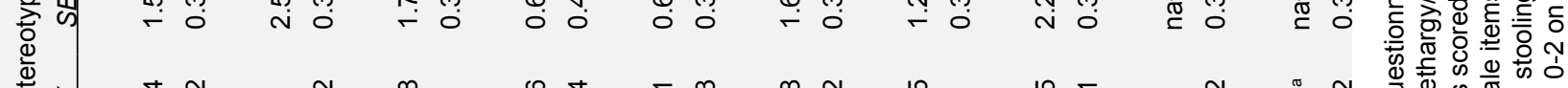

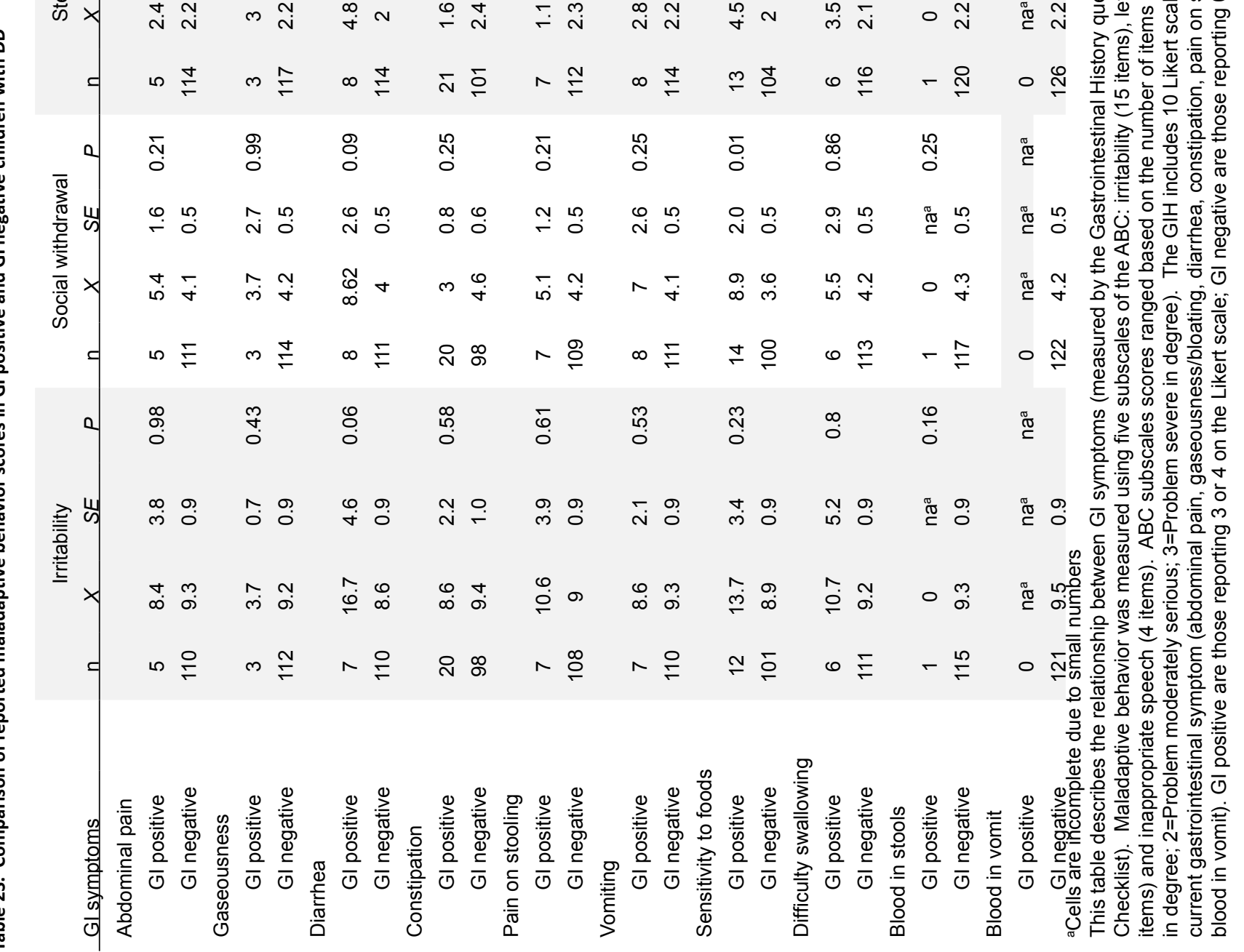

